\title{
When we can't get together: how large is the value added of video-conferencing over computer-conferencing
}

\author{
J. Rohrbaugh, University at Albany, SUNY, U S A, Chair \\ C. Csaki, Bell-Northern Research Ltd, Canada \\ P. Danyi, Technical University of Budapest, Hungary \\ W.G. Hewett, Deakin University, Australia \\ S. Midkiff, Virginia Tech, USA
}

\section{INTRODUCTION}

When group members are unable to work together in the same room at the same time, there are at least five options currently available. The first is to wait as long as it takes until they can. The second is to add and subtract names from the original membership list to create a new group with one, overriding qualification: mutually convenient schedules. The third is to assign the responsibility to a smaller subgroup who can manage to be in one room at one time; at the extreme, this means one person can make all the key decisions. The fourth and fifth options are the subject of this panel.

Considerable developmental work on videoconferencing (option four) and computer conferencing (option five) has occurred over the past three decades, but it is only in the 1990s that communication networks have developed to the point that they can be used with relatively greater ease. Videoconferencing increasingly has been designed to simulate the intimacy and directness of face-toface meetings, as if everyone were in the same room at the same time, for participants who actually may be thousands of miles away. Computer conferencing supports any time, any place meetings in which the interaction process is structured to completely connect all participants but without the immediate, give-and-take exchange of messages.

The proposed panel is prepared to address the question, "How large is the value added of videoconferencing over computer conferencing?" Two large and complex sets of issues will be examined during the session. First, the situational context of meetings may influence the appropriate selection of technology. Second, the resources expended to convene alternative forms of conferencing need to be considered. These two sets of issues, taken together, allow for an explicit accounting of the "value added" of videoconferencing. 


\section{SITUATIONAL CONTEXT}

The situational context can be identified by
a) the organizational conditions
b)the problem domain
c) the meeting purpose.

Organizational conditions may include (but certainly are not limited to) the leadership and decisionmaking style to which the group is accustomed. The problem domain refers to the many dimensions that distinguish one type of problem from another (e.g., structured versus unstructured or creativity versus choice-making tasks). A meeting's purpose is defined by the limited or extensive goals (and associated objectives) that its convenors have established to define its success. Depending on organizational conditions, problem domain, and meeting purpose, the value added of videoconferencing over computer conferencing will vary.

\section{RESOURCES EXPENDED}

The resources expended include
a) communication infrastructure
b) computer hardware/software
c) start-up support

Resources devoted to communication infrastructure might comprise such categories as facility expenses (including non-computer equipment) and network costs. Hardware/software costs depend upon the extent to which an organisation must acquire additional computer technology beyond that which currently is available. Start-up support covers a broad array of training and facilitating services required to initiate first-time users. Depending on the system demands for communication infrastructure computer hardware/software, and start-up support, the value added of videoconferencing over computer conferencing will vary

\section{PANEL FORMAT}

After some introductory remarks from the panellists, the chair will raise a number of questions (see Table 1) as a prelude to open discussion. 
Table 1 questions to motivate interventions and discussions:

Q1 Are there types of organizational conditions (e.g., leadership and decision-making styles) that are better suited to videoconferencing or computer conferencing?

Q2 Are there circumstances (e.g., crisis) that are better suited to one or the other?

Q3 What types of decision situations can be attacked efficiently?

Q4 What about group dynamics and team-building? How can they be supported?

Q5 How are different meeting goals supported?

Q6 What computing and communication infrastructure are required, and what is the availability of them?

Q7 What forms of communication can be supported, e.g. text, graphics, pictures? How easily?

Q8 What are the costs? Fixed costs (hardware, software, services) and variable costs (communication time, facilitator's time, etc.)?

Q9 What kind of preparation (including physical and mental) are the meeting participants required to make? And how much preparation is necessary on the facilitator's side?

Q10 What about first-time users? Can they use the system right away or do they need special training? How much? 